\title{
CREACIÓN Y DEVOCIÓN EN CANCIONEROS CATALANES: EL CANÇONER SAGRAT DE VIDES DE SANTS
}

\author{
Tomàs MARTínez RoMero \\ Universitat Jaume I
}

\section{Entre la creación y la devoción: algunas reflexiones teóricas a propósi- to de los cancioneros religiosos}

No resulta ninguna novedad afirmar que los cancioneros religiosos no han tenido un trato semejante a los de tema profano. Recientemente, a propósito de las Cantigas de Santa María, Valeria Bertolucci ${ }^{1}$ se quejaba de la desatención general hacia la poesía lírica no profana, y más en concreto hacia la organización de sus materiales. De hecho, es lícito afirmar sin ningún reparo que, a pesar del volumen considerable de obras existentes, la literatura religiosa es uno de los ámbitos peor estudiados de nuestra cultura medieval. Así lo declaraba también, hace ya más de veinte años, Albert Hauf ${ }^{2}$, sin duda alguna uno de los mayores especialistas en este ámbito. Al margen de la mayor o menor adhesión personal, del gusto o del disgusto del investigador, este asunto ha merecido, objetivamente, menos páginas críticas de las que debiera. No hablo, claro está, de aquellas dedicadas a obras concretas de autores significativos, valoradas en tanto que piezas cultas que encajan en una trayectoria literaria y vital de un escritor, sino de los cancioneros en cuanto tales, amalgama física que puede obedecer y obedece a múltiples causas. El cancionero religioso no despierta pasiones en la actualidad, aunque en su momento fuera otra su repercusión, mucho mayor. Independientemente de

\footnotetext{
${ }^{1}$ Valeria Bertolucci, «Libro di autore e libro di autori: il caso delle Cantigas de Santa María», en Canzonieri iberici, ed. Patrizia Botta, Carmen Parrilla, Ignacio Pérez Pascual, A Coruña, Editorial Toxosoutos - Università di Padova - Universidade da Coruña, 2001, 3 vols., I, pp. 125-137: «La ricerca sull'organizzazione delle grandi sillogi medievali di poesia lirica, ha riguardato e riguarda quasi esclusivamente il versante "profano" di tale produzione in versi», p. 125.

${ }^{2}$ Albert Hauf, D'Eiximenis a sor Isabel de Villena, Aportació a l'estudi de la nostra cultura medieval, València - Barcelona, Institut de Filologia Valenciana - Publicacions de l'Abadia de Montserrat, 1990, pp. 19-20: «és una de les parcel·les més mal estudiades de la nostra història cultural».
} 
la valoración, su análisis topa, además, con otras dificultades, al menos en lo que respecta a la realidad valenciana, sobre la cual pretendo reflexionar aquí. La primera, desde luego, la de configurar y delimitar su espacio propio, para distinguirlo de otros productos como los volúmenes de poesías de certamen o los libelli precum ${ }^{3}$, devocionarios, oracionales o himnarios. En el primer caso - es decir, en el libro manuscrito o impreso que recoge las contribuciones fruto de un encuentro literario- parece claro que la limitación del tema a la glosa de un santo o santa, a la loa mariana o a alguna de sus advocaciones, o a algún aspecto de la pasión y muerte de Cristo, determina que el resultado final tenga un carácter monográfico, distintivo respecto a otras muestras literarias. Además se incluyen en él, a modo de textos de acompañamiento, fragmentos que dan noticia del inicio y de la finalización del concurso. Un recorrido somero por el libro de Antoni Ferrando sobre los antiguos certámenes literarios valencianos conocidos ${ }^{4}$, todos ellos religiosos, confirma este extremo. Sin embargo, -y esto es lo que más nos interesa- no siempre se copian en bloque todas las colaboraciones de un certamen ni estas tienen una difusión homogénea. Solo cabe recordar la obra de mosén Tallante («Por ser tan preclara la más que perfeta») recogida en la primera edición del Cancionero general y ya presentada anteriormente en el certamen inmaculista convocado por Ferrando Díeç en 1486. O mejor aún, las poesías de Vicent Ferrandis incluidas en la segunda edición de la recopilación de Hernando del Castillo y ganadoras de encuentros literarios en Valencia ${ }^{5}$. A lo que parece, el hecho de que estos eventos no tuvieran su reflejo en una publicación propia contribuyó sin duda alguna a que Castillo, atento a estos asuntos de imprenta, decidiera incorporarlas a su magna obra. La gran ventaja es que allí, en el Cancionero general, se explicita la procedencia y la autoría, lo que no siempre ocurre, ni por asomo. Al cabo, si no fuera por esto, difícilmente acertaríamos a identificar algunas piezas copiadas al lado de otras de muy diferente género y tipología. Piénsese, por ejemplo, en la obra de mossén Bartomeu Dimas, ganadora de un certamen inmaculista en prosa catalana (1487), pero publicada en un incunable al lado de un sermón de Felip de Malla y de la valenciana prosa que

\footnotetext{
${ }^{3}$ A pesar del tiempo transcurrido, siguen siendo de referencia los textos editados por D.A. Wilmart, OSB, Precum libelli quattuor aevi Karolini, Roma, Ephemerides Liturgicae, 1940.

${ }^{4}$ Antoni Ferrando, Els certàmens poètics valencians dels segles XIV al XIX, València, Institució Alfons el Magnànim-Diputació de València, 1983.

${ }^{5}$ Fuera del ámbito valenciano, pero todavía dentro del Cancionero general, es sabido que la edición sevillana de 1535 reproduce el texto de algunas piezas devotas que provienen de actos literarios celebrados en esta ciudad andaluza, lo que da idea de lo común del fenómeno. Véase una aproximación somera a la poesía religiosa - castellana- de la compilación de Castillo en Olga Perotti, «La poesía religiosa en el Cancionero general de 1511», en I canzonieri di Lucrezia / Los cancioneros de Lucrecia, ed. de Andrea Baldissera, Giuseppe Mazzocchi, Padova, Unipress, 2005, pp. 247-262.
} 
nos propone Joan Roís de Corella en su Vesió. Ciertamente, solo la inclusión del «llibell» y del «introit», juntamente con la «sentència», sitúan la obra de Dimas en su sitio y nos certifican su fuente ${ }^{6}$.

$\mathrm{Y}$ es que puede resultar relativamente lógico que, en un cancionero religioso -también en uno profano-, se incluyan algunas piezas provenientes de encuentros literarios sin necesidad de declararlo. De hecho, el esquema métrico seguido o la preferencia por un determinado tratamiento o enfoque han sugerido en más de una ocasión la posibilidad de que tal o cual obra copiada en un cancionero se pudiera relacionar con tal o cual certamen. En este sentido, por ejemplo, se han señalado los paralelismos formales y temáticos de obras de certamen del siglo Xv valenciano con composiciones que transmite el conocido como Cançoner sagrat de vides de sants ${ }^{7}$, compilación marcadamente hagiográfica sobre la que me detendré después. Debemos distinguir, por tanto, lo que es un todo monográfico de lo que es un aprovechamiento ocasional de una parte en otro volumen concebido de manera diferente, por otros motivos y con objetivos distintos. Es en este último caso cuando se relajan las fronteras entre poesía de certamen y poesía de cancionero. Ahora bien, a diferencia de la poesía de certamen, en los cancioneros religiosos se debe buscar el espíritu cohesionador, su razón de ser, que en el otro caso ya se da en origen, con elementos como el cartel y la sentencia resolutiva, altamente identificadores de su procedencia.

Muy diferente es la relación que se establece entre cancioneros religiosos y libelli precum, libros de oraciones y devocionarios. Ahora debemos tener en cuenta, además, un elemento ausente en la comparación con los certámenes literarios: la potencial presencia de traducciones de composiciones latinas. No se trata de un asunto menor. El general desconocimiento por parte del investigador actual de los materiales litúrgicos y de devoción de la época medieval $-\mathrm{y}$, por tanto, la incapacidad de detectar las fuentes- provoca a menudo que pase como original en lengua románica alguna pieza, fundamentalmente anónima, que no es sino la traducción de lo que en su día fue una oración, un himno o cualquier otro producto. Con ello damos carta de naturaleza y de «genuinidad» literaria a lo que es una realidad de la práctica religiosa, aun reconociendo que, en este asunto que tratamos, las fronteras entre literatura y religiosidad son muy permeables y sus relaciones ofrecen

\footnotetext{
${ }^{6}$ Ferrando, Els certàmens, ob. cit., pp. 535-555.

${ }^{7}$ Ibídem, p. 139: «Diversos poetes anònims de certamen podrien haver tingut alguna cosa a veure amb aquests cançoners. Més avant tindrem l'avinentesa d'examinar l'estret paral·lelisme formal -mètric i estilístic-i, en alguns casos temàtic, entre algunes de les composicions del Cançoner Sagrat i d'altres que pertanyen a diversos certàmens coneguts de l'època».
} 
una casuística generosa. Por otra parte, no es del todo extraño encontrar cancioneros en los que se combinen obras de tradición culta latina más o menos canónicas o de autoría reconocida con otras poesías romances de génesis y difusión popular, a veces incluso con el añadido de la traducción en prosa de alguna composición breve, si el espacio lo permite.

Un ejemplo prototípico de lo que vengo diciendo es, claro está, el denominado Cançoner espiritual, actualmente repartido entre los folios de los manuscritos 1494, 1579 y 1682 de la Biblioteca de Catalunya, aunque inicialmente fuese un solo volumen ${ }^{8}$. Efectivamente, Ernest Moliné i Brasés publicaba hace un siglo un artículo con la edición de las obras catalanas contenidas en este Cançoner (no así las latinas que las acompañaban) ${ }^{9}$. Por su contenido, propio de una práctica personal e individual, el editor apuntaba a un origen relacionable con «mans consagrades». Lo cierto es que la parte latina ofrecía un notable repertorio de himnos como «Virgo plorans filium», «Gaude virgo mater Christi» o «Sancta Dei genitrix», de temática mariológica, copiados al lado de poemas como «Ave Maria yoiosa», «En lo món, pus fos dotade» o «Déus te sal, regina», que son goigs o llaors de claro carácter popular, hasta el extremo de ser recogidos por un investigador tan prestigioso en esta materia como Josep Romeu i Figueras ${ }^{10}$. Es decir, se reunían en un mismo espacio obras canónicas cultas y composiciones creativas. El caso es que, en este cancionero espiritual, se introdujeron también, en catalán, las conocidas misas de san Amador, algunas canciones navideñas o una traducción del De duodecim diebus veneris atribuido a Clemente de Roma ${ }^{11}$. Es evidente que el único ingrediente común de todas estas piezas era el argumento religioso y que los materiales se acumularon sin más en el volumen original, de pequeña factura, tal como corresponde a los libros de devoción. La temática religiosa del Cançoner espiritual se debe entender, todavía, en sentido lato, puesto que se recogen en él hasta oraciones e invocaciones contra el mal tiempo, en latín y en catalán. Con todos estos elementos en juego, es lícito preguntarse en qué medida este Cançoner es realmente eso, un cancionero religioso, y en qué otra se trata de un libro de devoción, para uso personal, como su mismo

\footnotetext{
${ }^{8}$ Para todo lo que concierne a esta obra y a su composición, remito directamente a Tomàs Martínez Romero, «El Cançoner espiritual de Moliné i Brasés: una proposta de reconstrucció», Estudis Romànics, 34 (2012), pp. 419-429.

${ }^{9}$ Ernest Moliné i Brasés, «Textes vulgars catalans del segle xv», Revue Hispanique, 28 (1913), pp. 396-441.

${ }^{10}$ Josep Romeu i Figueras, Corpus d'antiga poesia popular, Barcelona, Barcino, 2000, pp. 137-150.

${ }^{11}$ Tomàs Martínez Romero, «Tradició catalana medieval del De duodecim diebus ueneris atribuït a Climent de Roma. Contribució a l'estudi de les traducciones quotidianes d'obres religioses breus», Anuario de Estudios Medievales, 41:1 (2011), pp. 291-309.
} 
formato muestra. Me temo que hay razones para defender ambas posturas, que no son ni deben ser completamente excluyentes. Al fin y al cabo la devoción no tiene por qué ser incompatible con la creación, ni al contrario. Solo es necesario reconocer perfectamente, en fin, los materiales y los objetivos con los que trabajamos y dar a cada cual el tratamiento que merece.

Sin duda alguna, un elemento importante de cara a validar el resultado final de una investigación sobre los cancioneros religiosos ha de ser la comprobación y la concreción -más allá de los lugares comunes- de las fuentes, saber si realmente lo que leemos corresponde a una creación autónoma o si, por el contrario, debemos asumir una determinada filiación, sea por vía de traducción o de recreación. En este último caso, tenemos que precisar todavía el proceso de transferencia, es decir, los mecanismos que eligió el autor para individualizar su obra respecto a su patrón de base. Aun reconociendo las dificultades de delimitar los espacios de creación en los siglos medievales, $\mathrm{y}$ la importancia del seguimiento de los modelos textuales como paso iniciático de la formación escolar, se me antoja imprescindible conocer qué hay de genuino en una obra, incluso aceptando la permeabilidad de géneros y sus íntimas conexiones a la hora de enfocar la valoración ${ }^{12}$. En esta ocasión, nos puede servir de muestra el análisis de las composiciones hagiográficas de los cancioneros religiosos y sus dependencias de la Legenda aurea ${ }^{13}$, sobre todo porque no es difícil reconocer la huella de esta en muchas obras anónimas o de perfil tradicional, moldeadas frecuentemente a partir de la acumulación de sensibilidades diversas. En el ámbito valenciano, este camino nos conduce inevitablemente al modelo de los goigs, no como entidad vinculable temáticamente al espíritu mariano -en la dirección de los goigs del papa Clemente IV, «Los VII gautz de nostra Dona»-, sino en tanto que formulación de un género popular en loor de María o de los $\operatorname{santos}^{14}$.

${ }^{12}$ Forzando al máximo las relaciones, se podrían analizar incluso hipotéticas vinculaciones entre coplas religiosas y predicación, no a nivel de formalización, sino en cuanto mecanismos de difusión de determinados valores, con voluntad potencial o explícita de influir sobre los destinatarios.

${ }^{13}$ Aunque, evidentemente, no es el único modelo hagiográfico medieval, sí que parece ser uno de los más productivos. Claro está que, a medida que retrocedemos en el tiempo, las influencias se muestran más opacas a nuestros ojos. Recientemente, por ejemplo, M. von Albrecht nos ha remitido al Séneca trágico y al Ovidio de las Metamorfosis como fuentes literarias del martirio de san Hipólito descrito por Prudencio (Michael von Albrecht, «Momenti della presenza di Seneca nella tradizione cristiana», en Seneca e i Cristiani, ed. Antonio P. Martina, Milano, Vita e Pensiero, 2001, pp. 5-39).

${ }^{14}$ Véase Dominique de Courcelles, Les histoires des saints, la prière et la mort en Catalogne, París, Publications de la Sorbonne, 1990, y «De la relecture à l'interprétation de vies saintes en Catalogne», en Miscel-lània Joan Fuster, ed. Antoni Ferrando y Albert Hauf, Barcelona - València, Publicacions de l'Abadia de Montserrat, 1991, vol. IV, pp. 147-163. 


\section{El Cançoner sagrat de vides de sants, ¿cancionero intencional?}

Efectivamente, Jordi Rubió, en las páginas referidas a la literatura catalana de la Historia general de las literaturas hispánicas de Díaz Plaja, señalaba como «les dues col·leccions més importants de poesies religioses en forma de goig» los dos volúmenes del Cançoner sagrat de vides de sants y, ya impreso en el siglo XVI, «el cançoner del poeta valencià Miquel Ortigues (1. ${ }^{\mathrm{a}}$ ed. a València, 1512, i després molt reimprès de manera total o parcial) $\rangle^{15}$. Se trata de cancioneros que se alejan, por su arquitectura y objetivos, de los compuestos por piezas más complejas o de autor conocido, como las castellanas contenidas en el de Ramón de Llavia o algunas de las composiciones cultas valencianas incluidas en el Nazareno, volumen facticio conservado en la Biblioteca Històrica de la Universitat de València. De las dos colecciones apuntadas por Rubió, el Cançoner sagrat de vides de sants, todo él en catalán, tiene el mérito de permitirnos analizar con detalle algunos problemas que se relacionan directamente con lo expuesto anteriormente, no solo sus relaciones con fuentes como la Legenda aurea (que muy a menudo se han dado por supuestas), sino también su independencia -o no- respecto a los certámenes literarios y a los libros de argumento devoto.

El contenido del Cançoner sagrat, casi monográficamente hagiográfico, nos ha llegado en dos manuscritos copiados por la misma mano en la segunda mitad del xv: el manuscrito 3 de la Biblioteca de Catalunya y el 6519 de la Biblioteca Serrano Morales, del Ayuntamiento de Valencia ${ }^{16}$. De hecho, parece que, si no era así desde el inicio ${ }^{17}$, durante el siglo XVIII ya se componía de estos dos volúmenes, que pertenecieron al erudito valenciano Gregori Mayans i Siscar. En el siglo XIx, el librero Pere Salvà tuvo en su poder el primer volumen, el de la Biblioteca de Catalunya, que pasó a esta institución en 1907 ${ }^{18}$; fue el propio Salvà quien lo denominó Cobles fetes en laors de diversos sants. Lo que no cabe duda es que el nombre por el cual es conocido hoy el conjunto de los dos códices, Cançoner sagrat de vides de sants, fue otorgado por Massó Torrents, quien lo editó completo, juntamente

\footnotetext{
${ }^{15}$ Cito de la traducción catalana: Jordi Rubió i Balaguer, Història de la literatura catalana, I, Barcelona, Publicacions de l'Abadia de Montserrat, 1984, p. 451.

${ }^{16}$ Remito a BITECA, manid 1328 y 1329, respectivamente, donde se aporta además la bibliografía de referencia. El manuscrito valenciano no tiene foliación.

${ }^{17}$ Hay quien piensa, con argumentos razonables, que quizá nunca hubo un único códice que contuviese lo que hoy son dos volúmenes. Véase Sergi Barceló Trigueros, El Cançoner sagrat de vides de sants (Poesia valenciana religiosa de la baixa Edat Mitjana), Treball d'investigació tutelat per la Dra. Marinela Garcia Sempere, Departament de Filologia Catalana - Universitat d'Alacant, 2011, p. 44.

${ }^{18}$ Repertori de manuscrits catalans (1474-1620), vol. 1, dir. Eulàlia Duran, Barcelona, Institut d'Estudis Catalans, 1998, pp. 124-126.
} 
con Foulché-Delbosch, en 1912 ${ }^{19}$. Finalmente, en 1915-20, Ramon Miquel i Planas atribuyó la autoría de estos versos medievales al notario valenciano Miquel Ortigues ${ }^{20}$; a partir de entonces se han sucedido las opiniones a favor o en contra de dicha atribución, sin duda muy poco consistente hasta ahora ${ }^{21}$. Casi un siglo después, en 2011, Sergi Barceló Trigueros ${ }^{22}$ ha puesto al día todos los elementos de análisis que acabo de sintetizar ahora y los materiales necesarios para la publicación de una futura edición crítica del texto.

Lo primero que salta a la vista después de leer la nómina de composiciones del Cançoner sagrat es su lejanía respecto al orden de aparición de santos y santas en el calendario litúrgico cristiano, al menos tal como lo seguía la Legenda aurea más o menos canónica ${ }^{23}$. Tampoco es la única diferencia respecto a esta obra: el cancionero no tiene ninguna voluntad enciclopédica, ni incluso un claro e inmediato criterio de ordenación; además, tiene un formato muy alejado del in-folio que siguen -excepto una ${ }^{24}$ - todas las copias enteras o fragmentarias de la Legenda en catalán. El responsable del Cançoner tenía, por tanto, una clara consciencia de independencia respecto a ella y la idea de que su trabajo obedecía a otras reglas, lo que no implica que no

${ }^{19}$ Cançoner sagrat de vides de sants, ed. de Jaume Massó y Raymond Foulché-Delbosc, Barcelona, Societat Catalana de Bibliòfils, 1912, 2 vols.

${ }^{20}$ Ramon Miquel i Planas, «El Cançoner Devot, d'en Miquel Ortigues (Segle Xvi)», Bibliofilia, 2:12 (1915-20), cols. 1-55.

${ }^{21}$ Sergi Barceló promete nuevos argumentos a favor: «gràcies a les investigacions sobre fons històrics i documentals, m'atrevisc a dir que pertany al notari valencià Miquel Ortigues»; véase Sergi Barceló Trigueros, «L'estil i la llengua de Miquel Ortigues al Cançoner sagrat de vides de sants», Scripta, 2 (desembre de 2013), pp. 61-83, p. 64.

${ }^{22}$ Barceló, El Cançoner sagrat, ob. cit.

${ }^{23}$ Canónica en el sentido que crea un corpus más o menos estandarizado, sobre todo respecto a los legendarios anteriores, como ha mostrado Giovanni Paolo Maggioni, «Riletture e riscritture agiografiche del XIII secolo: i leggendari abbreviati», en Vides medievals de sants: difusió, tradició i llegenda, ed. Marinela García Sempere, M. Ángeles Llorca Tonda, Alacant, Institut Interuniversitari de Filologia Valenciana, 2012, pp. 11-34. Ello no evitó, por supuesto, la existencia de contaminaciones e irregularidades en el complejo proceso de difusión y de aclimatación a las realidades de los destinatarios, una aclimatación que no es privativa de la Legenda aurea estricta. Los objetivos, claro está, determinan en gran manera la tipología del producto. Recientemente, un estudio sobre el manuscrito Ripoll 113 de l'Arxiu de la Corona d'Aragó ha puesto de relieve las relaciones de causa-efecto entre la presentación de las 71 vidas que contiene (divididas en dos bloques, uno de santos y otro de santas) y el más que posible origen del códice en Santa María de Sigena, en Huesca, monasterio doble de la orden del Hospital de San Juan de Jerusalén (Glòria Sabaté, Lourdes Soriano, Gemma Avenoza, «Vides de sants en manuscrits incomplets i membra disiecta, o com completar el nostre coneixement del que foren les biblioteques antigues», en Vides medievals de sants, ob. cit., pp. 35-63).

${ }^{24}$ Se trata del códice 178 del Archivo de la Catedral de Barcelona, en octavo (véase Gemma Avenoza, Marinela Garcia Sempere, «Santos y santas en la tradición escrita catalana medieval», en De lo humano a lo divino en la literatura medieval: santos, ángeles y demonios, Granada, Universidad, 2012, pp. 47-60, p. 59). Como es bien sabido, el octavo es, con el cuarto, el formato ideal para los libros de horas, devocionarios y vidas concretas de santos. 
la tuviera como referente. Que no siguiese la macroestructura propuesta por Vorágine no implica ni desapego ni ninguneo: una cosa eran los materiales utilizados y otra muy distinta la disposición de estos y los objetivos finales del producto ya confeccionado. Ciertamente, aunque se prescinde de ella en más de una ocasión, parece ser que la Legenda -o la tradición dinámica que recoge la Legenda-constituye la fuente principal de las «historias» de santos que se relatan en el Cançoner, si bien se opta por imponer un punto de vista más realista y menos sobrenatural, de acuerdo con la voluntad de relatar los momentos más humanos y afectivos de los protagonistas ${ }^{25}$. De hecho, de las 25 composiciones del primer volumen (el de Barcelona), 24 de asunto hagiográfico o mariano más la Salve Regina, únicamente no están presentes en la Legenda las historias relativas a la virgen de Montserrat y a santo Tomás de Aquino. Ciertamente, la proporción cambia bastante en el segundo volumen, donde las vidas compartidas llegan a ser poco más de la mitad ${ }^{26}$. La explicación es fácil: en la parte final se encuentran unas cuantas composiciones no estrictamente de santos, como las consagradas a los reyes de Oriente o a Adán y Eva, además del Credo y los versos dedicados a la Virgen de la Piedad, a la aparición de Cristo a María, al Santo nombre de Cristo o a la Vera Cruz. Se trata, por tanto, de estrofas que no responden a los argumentos que esperaríamos encontrar en un Cançoner sagrat si este se pretende que sea solo de vides de sants. Siendo así, tendríamos que pensar, en efecto, que el Cançoner se parece más a una especie de cancionero depósito, a una acumulación de pequeñas obras de temática religiosa en catalán, que a un repertorio ordenado y coherente de ellas; de hecho es difícil encontrar un hilo conductor que relacione los poemas hagiográficos más o menos previsibles con aquellos otros que lo son menos, y a todos estos con los versos no estrictamente hagiográficos que cierran el volumen ${ }^{27}$.

\footnotetext{
${ }^{25}$ Como afirma Barceló, «L'estil i la llengua», art. cit., p. 65. Habla de «humanisation du divin» Dominique de Courcelles, «De la louange collective a l'angoisse du salut individuel: étude du ms. 3 de la Bibliothèque de Catalogne a Barcelone», Mélanges de la Casa de Velázquez, 22 (1986), pp. 111-129, esp. pp. 117-118.

${ }^{26}$ Siempre comparando con la edición de Giovanni P. Maggioni (Iacopo da Varazze, Legenda aurea, 2 vols., Firenze, Sismel - Edizioni del Galluzzo, 1998) y obviando de alguna manera la flexibilidad de contenido que ofrecían las copias y traducciones de la Legenda.

${ }^{27}$ A pesar de ello, no debemos perder de vista que, en la obra de Vorágine, los capítulos dedicados a los santos se complementan con otros que tratan de fiestas temporales del ciclo litúrgico, incluidas las que tienen que ver con el culto de la Cruz o con el ciclo de Cristo o de la Virgen. No hay que descartar que el autor del Cançoner tuviese en cuenta el orden de presentación ofrecido por otros repertorios de santos, por ejemplo alguno de los incluidos en el "índice" hagiográfico de Fernando Baños (Las vidas de santos en la literatura medieval española, Madrid, El Laberinto, 2003). Se trata de una circunstancia que, en todo caso, tendrá que valorar el futuro editor de la obra.
} 
El problema es, a mi parecer, que seguimos analizando el contenido del Cançoner «solo» a partir del modelo de la Legenda, cuando hemos comprobado que su factura externa y sus objetivos desmienten una vinculación «estructural» con ella. Todo es mucho más fácil cuando prescindimos por un momento de las fuentes, de las referencias a Vorágine, y tratamos el conjunto del Cançoner sagrat en parte como un cancionero, en parte como libro que mantiene una función religiosa en su origen o en su destino; esto es, cuando aplicamos sobre el terreno las reflexiones anteriores sobre las relaciones entre los cancioneros religiosos y otros materiales. Ahora es cuando resulta fundamental subrayar que los versos del Cançoner destacan sobre todo el martirio, el sufrimiento, la pasión o la redención de los santos, y no los elementos sobrenaturales, los más milagrosos e impactantes. Cierto también que la narración poética de los méritos realizados por esos hombres y mujeres, su «pasión», enlaza con la literatura pasional, contemplativa y afectiva de entonces, promovida en gran manera por la religiosidad íntima y por el cristocentrismo de la devotio moderna ${ }^{28}$, aunque no debemos olvidar tampoco que la génesis de una buena parte de la literatura hagiográfica se encuentra ya en las acta y en las passiones de los santos ${ }^{29}$.

En gran parte, el conjunto de los poemas estrictamente hagiográficos del Cançoner seguía, en efecto, un común denominador argumental claro, que fijaba su atención en los tormentos, enfermedades, penalidades, penitencias, persecuciones o injurias padecidos por el santo o santa ${ }^{30}$, o bien que contenía algún tipo de referencia, ni que fuera de pasada, a la cruz, a los tormentos, a la muerte o a la pasión de Cristo ${ }^{31}$. Si descontamos de las 59 composiciones del Cançoner sagrat aquellas que se guían por estos parámetros y las oraciones finales de las que hablaremos después, solo nos quedan unas pocas que parecen no tener ningún criterio identificativo explícito: las consagradas a María Egipcíaca, Virgen de Montserrat, san Agustín, santo Domingo, san Basilio,

\footnotetext{
${ }^{28}$ Como bien señala Barceló, El Cançoner sagrat, ob. cit., pp. 24-25, 53. Por ello, no es extraño que el tema de la Pasión, de la contemplación y meditación sobre la muerte de Cristo, sea también el predilecto de las páginas religiosas del Cancionero general (Perotti, «La poesía religiosa», art. cit., p. 252).

${ }^{29}$ En esta misma dirección, juntamente con la práctica poética propia, conviene tener en cuenta la tradición latina medieval, punto de partida también de numerosas oraciones e himnos. Así se expresaba Dominique de Courcelles («De la louange collective», art. cit., pp. 115-16): «Les “joies”, et en particulier celles du manuscrit qui nous intéresse, sont tout à fait comparables par leurs thèmes hagiographiques aux hymnes latines composées pour les églises catalanes des siècles antérieurs».

${ }^{30}$ Martín, Jaime, Bartolomé, Tomás apóstol, Andrés, Esteban, Hipólito, Vicente mártir, Francisco, Bricio, Tomás de Canterbury, Felipe y Jaime, Marcos Evangelista, Simón y Judas, Bárbara, Tecla, Macián, Mateo, Bernabé, Dimas, Eulalia, Blas, Honorato, Pablo, Jorge, Antonio de Padua, Apolonia, Magdalena, Antonio, Sebastián, Juliana, Agnés, Margarita, Lucía, Adan y Eva.

${ }^{31}$ Juan Evangelista, Andrés, Gil, Francisco, Lucas, Marta, Tomás de Aquino, Dimas, Jorge, Magdalena, Margarita, Caterina de Siena, Adan y Eva.
} 
Lázaro, Macario, los Reyes de Oriente, Luis obispo, Amador y Guillermo. De este registro todavía podríamos restar los versos destinados a algún santo fundador (Domingo) y a los padres de la Iglesia (Agustín y Basilio). Por otra parte, no resulta sorpresiva la inclusión de la Virgen de Montserrat; tampoco la de los Reyes de Oriente, la de san Guillermo, muy valorado en tierras catalano-aragonesas ${ }^{32}$, de san Amador, bien conocido gracias al treintanario de misas por la redención de los condenados al Purgatorio ${ }^{33}$, o de san Luis obispo, relacionado con la ciudad de Barcelona. No nos costaría mucho encontrar aún algún elemento de la vida de los santos de esta lista relacionable más o menos tangencialmente con los otros. De hecho, Lázaro, por ejemplo, tiene una vinculación directa, familiar, con Marta y Magdalena (figura que resulta de la confusión de los personajes de María de Magdala, María de Betania y la pecadora arrepentida que lava los pies de Jesús). Puede, en fin, que no sea tan ocasional que la colección se abra con la historia de María Egipcíaca, patrona de los penitentes y de la castidad, cuando, en el Cançoner, hay tantas pruebas y modelos de sacrificios en busca de la virtud y de la salvación. Tampoco conviene descartar, en suma, la posibilidad de que la inclusión de algún poema obedeciera simplemente a razones de oportunidad, gusto o convención.

Nos faltarían por valorar todavía no tanto los perfiles argumentales, cuanto las reglas seguidas para la ordenación de las obras en el Cançoner ${ }^{34}$. Ahora bien, si consideramos que, en este, el tema del ejercicio de la virtud y de la imitación-modelo de Cristo se prioriza sobre cualquier realidad histórica, puede resultar hasta cierto punto lógico que las piezas poéticas conserven su identidad propia y se independicen de una cronología, de una sujeción a cualquier pauta del tiempo litúrgico, que ahora no interesa ni como criterio de clasificación, ni como modo de presentación ${ }^{35}$. Cada una de las «historias» de santos empieza y termina en sí misma, sin ninguna otra relación con la siguiente que la común exhibición de experiencias humanas de ejemplaridad positiva. A partir de aquí, las composiciones se debieron de copiar teniendo

\footnotetext{
${ }^{32}$ Guillermo es, en algunas fuentes, protagonista destacadísimo de la conquista de Barcelona para los francos y, por tanto, responsable de su pertenencia a la Europa cristiana: véase Hèctor Càmara, ${ }^{2}{ }^{a}$ Ángeles Llorca, «Relatos hagiográficos de un héroe épico: Guillermo de Tolosa», Cuadernos de Investigación Filológica, 37-38 (2011-2012), pp. 61-96.

${ }^{33}$ Jordi Cerdà Subirachs, Les misses de sant Amador: purgatori i cultura popular, Barcelona, Publicacions de l'Abadia de Montserrat, 2006.

${ }^{34}$ Para el desarrollo de este aspecto, sigo las fundamentales orientaciones teóricas y prácticas de Vicenç Beltran, «Tipología y génesis de los cancioneros. La organización de los materiales», en Estudios sobre poesía de cancionero, A Coruña, Toxosoutos, 1999, pp. 9-54; íd., «The Tipology of Cancioneros: Compiling the Materials», en Poetry at Court in Trastamaran, ed. Michael Gerli, Julian Weiss, Tempe, 1998, pp. 19-54.

${ }^{35}$ De Courcelles, «De la louange collectiva», art. cit., p. 128.
} 
en cuenta los materiales a disposición y siguiendo las necesidades del espacio limitado por los cuadernos. Porque parece quedar fuera de discusión que el Cançoner se configura a partir de la unión de cuadernos completos ${ }^{36}$, de la misma manera que otros cancioneros catalanes, como el Vega-Aguiló o el Jardinet d'orats, siguiendo una técnica bien conocida en la tradición occitana. Esa dinámica de trabajo determinó con mucha probabilidad el orden de aparición de las estrofas del Cançoner sagrat y fue la causa también de los pequeños desajustes que podemos apreciar, como intentaré demostrar inmediatamente. El autor de la selección no obedecía, pues, a la tradición de los legendarios, sino a motivaciones religiosas más íntimas y a prácticas en absoluto ajenas a la confección de cancioneros.

Desde estos parámetros debemos explicar igualmente, en principio, la rareza de encontrar un último cuaderno con oraciones y composiciones de estructura y características muy diferentes a las precedentes. En él leemos, efectivamente, Lo Credo in Deum, Cobles fetes en laor dels gloriosos nostre pare Adam y nostra mare Eva, Cobles fetes en laor de la gloriosa Verge Maria de Pietat ab set virtuts contràries als set peccats mortals, Cobles fetes sobre la aparició que féu nostre Senyor Déu, Cobles fetes en laor del sant Nom de Jesús y Cobles fetes en laor de la santa Vera Creu. No cuesta mucho comprobar que, de las seis obras, las tres últimas se centran en elementos relacionados con la muerte de Cristo y la cruz, es decir, con argumentos que leemos en muchos versos hagiográficos anteriores, con los que guardan, pues, una relación evidente. Lo mismo ocurre con las coplas dedicadas a la Virgen María de la Piedad, pero ahora sobre la base del concepto de «virtud», también de amplio recorrido en el Cançoner. Por otra parte, el Credo, por sus mismas características, podía y puede ejercer una función de comodín, apto para cualquier contexto religioso. No ocurre así con los versos destinados a Adán y Eva, que, aunque no estrictamente hagiográficos, conservan una estructura semejante a ellos; se trata de una circunstancia que se repite en la obra dedicada a los tres Reyes de Oriente, con la diferencia de que esta sí que está incluida en un cuaderno con composiciones consagradas a las vidas de santos $^{37}$. Creo sinceramente que el poema de Adán y Eva está situado aquí, en este último pliego, por razones que nada tienen que ver con la temática o con la estructura, y sí con la utilización del espacio físico de los folios.

\footnotetext{
${ }^{36}$ Barceló, El Cançoner sagrat, ob. cit., p. 47: «El volum [de la Serrano Morales] consta de sis quaderns que han estat copiats independentment $i$ ajuntats al remat. Ho podem deduir perquè cada quadern presenta un nombre de sants i cap composició ultrapassa el seu quadern».

${ }^{37}$ El cuarto del mismo manuscrito de Valencia. Véase un cuadro con la estructura de todas las composiciones hagiográficas -incluidas las coplas dedicadas a Adán y Eva y a los Reyes de Oriente- en Barceló, El Cançoner sagrat, ob. cit., pp. 58-59.
} 
Todavía existe otro elemento que me induce a pensar tanto que el cuaderno final se copió independientemente, como que tenía una función especial en el conjunto del Cançoner. Así es. El autor material del cancionero había elegido siempre para sus composiciones títulos muy descriptivos, con el nombre del personaje al que se hacía referencia o al tema del que se trataba. Si en este caso decidió dar el título de Lo Credo in Deum ordenat en pla y obres rimades a la obra con la que arrancaba el pliego fue porque, efectivamente, copiaba el Credo y otras obras rimadas, las que componían el resto del cuaderno, pensado como un bloque homogéneo. Lo que cabe preguntarse es si ese conjunto se ideó así desde el inicio, como parte esencial del Cançoner. Nuevamente tenemos que ir a la literatura religiosa y a los cancioneros, y no a los legendarios medievales, para explicarlo. En efecto, estas estrofas contribuyen a dar al volumen -volúmenes- un aire más devoto y contemplativo al final, justo en el lugar destinado a las oraciones de cierre en obras de cierta envergadura $^{38}$. Pero es que también, de alguna manera, se reproducía a nivel macroestructural un esquema parecido al que presentaban las historias hagiográficas, con su loa-narración, completada o no con una «tornada» y finalizada con una oración. Solo que ahora la narración era el conjunto de vidas de santos copiadas en los doscientos folios anteriores y la oración, no una, sino todo un cuaderno, el final. El compilador del Cançoner, fuera o no el autor literario de todo él, ya tenía in mente la organización de todo este material desde su primerísima concepción y únicamente introdujo algunos leves retoques, más por razones coyunturales, de espacio físico, que conceptuales. Veamos, finalmente, en qué consistieron estos retoques y por qué se produjeron algunas pequeñas distorsiones respecto a lo que podríamos considerar el plan inicial de trabajo.

\section{De distorsiones y reintegraciones en el Cançoner sagrat}

Sin duda alguna, el responsable del Cançoner sagrat quería que todas las composiciones empezaran en el recto del folio y que ninguna se extendiera más allá de su cuaderno de inicio. Se trata de un detalle que tiene muy en cuenta y un argumento más -la presentación- para pensar en un destinatario ajeno a él. De hecho, lo normal es que el verso quede vacío si la composición anterior no llega a ocuparlo. Eso ocurre en todas las «cobles» excepto en cinco, que empiezan en el verso. En el primer volumen del Cançoner, el de la Biblioteca de Catalunya, sucede en las coplas dedicadas a san Martín, en el

${ }^{38}$ Hecho que indica convenientemente Barceló, El Cançoner sagrat, ob. cit., p. 53. 
primer cuaderno (f. 8v), y en las coplas en loor de san Agustín y en la Salve Regina, en el segundo (ff. 25v y 29v). En el primer caso, las coplas a san Martín van flanqueadas por caras en blanco (ff. $8 \mathrm{r}$ y $12 \mathrm{v}$ ), por lo que cabe suponer que el comienzo en verso de folio tiene que deberse a un simple descuido o, más probablemente, al deseo de no dejar un folio entero en blanco, criterio que se mantiene escrupulosamente en todo el Cançoner y que se hubiera alterado si se hubiera empezado a escribir en el 8r.

Primer cuaderno de BC 3:

f.1r. $\quad$ Cobles fetes en laor de senta Maria Egipciaqua

f.4r. Cobles fetes en laor de la gloriosa Verge Maria intitulada de Montserrat

f.8r. En blanco

f.8v. Cobles fetes en laor del confessor e bisbe sent Martí

f.12v. En blanco

En el caso de los versos dedicados a san Agustín y a la Salve Regina, sí que parece que hay argumentos para suponer que el copista fue plenamente consciente de su comienzo en el verso: la inclusión de las dos composiciones se tenía que ajustar necesariamente al espacio que restaba del cuaderno, es decir, a once caras.

Segundo cuaderno de BC 3:

f.13r. Cobles fetes en laor del gloriós sent Johan Evangeliste

f.17r. $\quad$ Cobles fetes en laor del beneyt sent Gil

f.21v. En blanco

f.22r. Cobles fetes en laor del gloriós apòstol sent Jaume

f.25v. Cobles fetes en laor del gloriós sent Agostí

f.29v. La Salve Regina ordenada en cobles.

Es cierto que hubiera podido empezar las coplas inmediatamente anteriores, las de san Jaime, en el folio 21v y así dejar el recto del folio 25 y del 29 para el inicio de las dos composiciones que cierran el cuaderno. Sin embargo, entra dentro de lo razonable que siguiera la dinámica de trabajo que se había fijado y, después de reproducir las estrofas dedicadas a san Jaime, se planteara cómo distribuir los materiales para ocupar el espacio que quedaba libre. Si comenzaba a transcribir los versos a san Agustín en el folio 26r, no cabía la Salve y le quedaba un folio entero en blanco (el 30), circunstancia que, como se ha señalado anteriormente, se intentó evitar siempre; si, por el contrario, 
se decidía por iniciar la copia en el verso, como finalmente hizo, le quedaban tres caras por rellenar $(29 \mathrm{v}, 30 \mathrm{r}, 30 \mathrm{v})$, justo la dimensión de la Salve Regina, que con toda probabilidad no tenía que ocupar este lugar originariamente. De hecho, era la única composición del Cançoner sagrat de tan pequeña dimensión: 48 versos heptasílabos. Conviene tener en cuenta, además, que cada cara del Cançoner está pautada para contener 20-22 líneas, con lo que el conjunto de estas tres ofrecía un total de 60-66 líneas ${ }^{39}$, fueran estas escritas o simplemente espacios libres entre estrofas. Es lo que corresponde a la suma de los 48 versos, más los espaciados entre las doce estrofas de la composición y el título de la Salve Regina. Ninguna de las obras hagiográficas del Cançoner hubiera podido incluirse en este punto del segundo cuaderno. Tampoco ninguna de las oraciones del final, igualmente de mayor extensión. El responsable del manuscrito, por tanto, calculó exactamente la dimensión de las coplas para incluirlas en su sitio. De hecho, lo más lógico, conceptualmente hablando, hubiera sido encontrar la Salve en el cuaderno final; lo mismo, pero justamente al revés, cabría decir de la composición dedicada a Adán y Eva, cuya estructura se asemeja a las composiciones hagiográficas y que es la única del cuaderno final que no encaja en su contexto. Llegados a este punto, es lícito preguntarse entonces si no es posible que estas dos obras hubieran permutado sus posiciones iniciales a causa de problemas de espacio. Conviene ahora, pues, analizar el pliego final -sin foliar- que cierra el Cançoner, siguiendo el mismo razonamiento empleado para dar razón del segundo y teniendo en cuenta su composición y el lugar en el que se inicia cada obra.

Último cuaderno de BSM 6519 y del Cançoner:

r. $\quad$ Lo Credo in Deum

v. Cobles fetes en laor dels gloriosos nostre pare Adam y nostra mare Eva

v. En blanco

r. Cobles fetes en laor de la gloriosa verge Maria de Pietat

v. $\quad$ Cobles fetes sobre la aparició que féu nostre Senyor Déu Jhesuchrist a la sua beneyta mare

$\mathrm{v}^{40} \quad$ [Cobles fetes en laor del sant Nom de Jesús]

r. Cobles fetes en laor de la santa Vera Creu

${ }^{39}$ Dada la diversidad de medidas de estrofa del Cançoner y también la ausencia de un patrón de página a seguir, este tuvo que ser con seguridad el sistema utilizado para confirmar si podía caber o no la Salve.

${ }^{40}$ Comparto la opinión de Barceló, El Cançoner sagrat, ob. cit., nota a la edición, de que el que sería el folio 12 de este cuaderno de cierre, actualmente perdido, contendría el final de la Aparició en el recto y el inicio de las coplas dedicadas al nombre de Jesús en el verso. 
Los 115 versos que componen Adam y Eva suponen el equivalente a unas seis caras de folio, tres más que la Salve. Por tanto, suponiendo por un momento que la Salve se hubiese copiado aquí en lugar de Adam y Eva, como parece más coherente, se hubiesen liberado tres caras respecto a lo que tenemos ahora. En este hipotético escenario, hubiera sido posible comenzar en recto las Cobles sobre la aparició y las coplas sobre el nombre de Jesús y, además, se hubieran podido copiar sin estrecheces los versos de la oración final de las Cobles de la santa Vera Creu, no a raya tirada, como ocurre ahora, puesto que no hay lugar para más. Sin descartar otras posibles explicaciones, creo que esta da cumplida cuenta de las pequeñas «distorsiones» del Cançoner.

No fue la única ocasión, sin embargo, en que nuestro calígrafo tuvo problemas de espacio - de falta de espacio- y tuvo que agudizar su ingenio. En el primer cuaderno del manuscrito de Valencia -lo que vendría a ser la segunda parte del Cançoner- ahorró todo lo que pudo para que cupiesen las estrofas que pretendía reproducir; por eso inició la composición dedicada a santa Tecla en el verso del folio en el que concluía la obra anterior, la referida a santa Bárbara. No le valió de nada el esfuerzo, porque poco después, antes de copiar las coplas a san Bernabé, se dio cuenta de que necesitaba imperiosamente dos folios más para poder reproducir enteras estas y las compuestas en loor de Dimas. Esta vez optó por la vía rápida de adjuntar estas cuatro caras extra al cuaderno inicial de $11+11$, justo allí donde comenzaban los versos destinados a san Bernabé.

Primer cuaderno de BSM 6519:

r. Cobles fetes en laors del gloriós sent Pau, primer hermità

r. Cobles fetes en laor de la gloriosa senta Bàrbera

v. Cobles fetes en laor de la beneyta senta Tecla

r. Cobles fetes en laor del gloriós sent Macià

r. Cobles fetes en laor del gloriós apòstol y evangeliste sent Matheu

v. Verso del folio en blanco

r. Cobles fetes en laor del beneyt apòstol sent Bernabeu

r. $\quad$ Cobles fetes en laor del sant ladre appellat Dimas

A pesar de estos pequeños errores de encaje, subsanados de la mejor forma posible, el Cançoner sagrat de vides de sants se configura como un cancionero perfectamente planificado a nivel de macroestructura, aunque dentro de los dos grandes bloques que lo sustentan, el hagiográfico y el «oracional», se optara por aplicar criterios más laxos y prácticos. En qué medida el autor material fue también el responsable literario, el creador, de todos los versos que transcribe 
es cosa que excede los objetivos de este espacio, aunque tengo el convencimiento de que, como mínimo, una buena parte de ellos tiene otros orígenes.

Recibido: 20/06/2014

Aceptado: 11/09/2014

\section{$\cos$}

\section{CREACIÓN Y DEVOCIÓN EN CANCIONEROS CATALANES: \\ EL CANÇONER SAGRAT DE VIDES DE SANTS}

RESUMEN: Reflexiones sobre la importancia del análisis de los cancioneros religiosos y sobre las dificultades con las que puede tropezar su estudio: fundamentalmente, la detección de los modelos y fuentes de las obras que en ellos se contienen y la fijación de los límites que los separan de otros textos religiosos o de devoción. Se ejemplifican estos problemas en el llamado Cançoner sagrat de vides de sants, ordenado no al estilo de los legendarios, sino sobre la base de conceptos religiosos (el sufrimiento y la virtud de los santos, la cruz, la pasión y muerte de Cristo...), y articulado como un cancionero «intencional».

Palabras clave: Cancioneros religiosos. Cançoner sagrat de vides de sants. Hagiografía. Oraciones. Literatura catalana medieval.

\section{CREATION AND DEVOTION IN CATALAN CANCIONEROS: THE CANÇONER SAGRAT DE VIDES DE SANTS}

ABSTRACT: Reflections on the importance of the analysis of the religious cancioneros and the difficulties with which your study might come across: mainly, the detection of the models and sources of the works which are contained in them and the fixing of the limits that separate them from other religious texts. This problems are exemplified in the so called Cançoner sagrat de vides de sants, which is arranged not in the ways of the legendary but based on religious concepts (the suffering and the virtue of the saints, the cross, the passion and the death of Christ...), and constructed as an «intentional» cancionero.

Keywords: Religious cancioneros. Cançoner sagrat de vides de sants. Hagiography. Prays. Medieval Catalan literature. 